\title{
Will Genotyping Replace Serology in Future Routine Blood Grouping? - Opinion 2
}

\author{
Hein Hustinx Stefano Fontana Peter Gowland Christoph Niederhauser \\ Blood Transfusion Service SRC Berne, Switzerland
}

There are 30 blood group systems and more than 300 blood cell antigens currently known. The experience during the last years has shown that it will become increasingly more difficult to handle all these polymorphisms with serological methods alone. Furthermore, new blood cell antigens are being identified yearly. Therefore, the use of molecular genotyping to efficiently predict the presence of these antigens in blood donors and patients will be unavoidable.

Blood group DNA analysis in patients and donors can be used in the following situations:

i) managing the availability and usage of components of rare blood types by donor mass screening for high-frequency antigen-negative donors,

ii) typing of multiply transfused recipients,

iii) typing of transfusion-dependent patients with sickle cell anemia, thalassemia or other hematological diseases to facilitate the selection of units compatible for a broad spectrum of antigens or for unusual antigens,

iv) typing of patients with a strong direct anti-human globulin test (DAT),

v) typing of platelet and leukocyte antigens (HPA, HLA) of donors and patients,

vi) resolving $\mathrm{ABO}$ and $\mathrm{RhD}$ serological discrepancies in patients,

vii) identifying some of the $\mathrm{RhD}$ variants in blood donors that are at risk for anti-D-alloimmunization,

viii) determining $R H D$ zygosity,

ix) confirming the true genotype when an antigen is weakly expressed (e.g. Del, Fy $^{\mathrm{x}}$ )

$\mathrm{x}$ ) genotyping donors for antibody identification panels, and

xi) fetal blood group diagnosis in maternal blood.

In addition, the lack of suitable immunohematological reagents favor the use of molecular methods. For instance, polyclonal antibodies are either not available or are too expensive, whereas monoclonal antibodies are often not able to detect all the variants.

\section{KARGER}

Fax +497614520714

Information@Karger.de

www.karger.com (c) 2009 S. Karger GmbH, Freiburg

Accessible online at:

www.karger.com/tmh
In Switzerland, the present routine blood group testing for red blood cells (RBCs) relies primarily on serological tests, such as serological antigen testing, antibody screening and cross-matching. In addition to the above mentioned indications in patient diagnostics, we envisage the use of genotyping in the future in two main directions. On the one hand to identify donors with rare blood groups, by performing a broad spectrum donor genotypic screening, and on the other hand by performing a similar extended typing of patients with the aim of obtaining more systematically compatible blood products for a broad spectrum of antigens. This would limit the number of alloimmunizations that often occur with the present transfusion practices. In particular, if patients could be transfused with compatible blood for the blood groups Rhesus, Kell, Duffy und Kidd, it would be expected that over $90 \%$ of the current alloimmunizations would be avoided [1,2]. The current molecular biological assays need to be improved, however, to the extent that donors and patients can be both efficiently and economically genotyped in a routine setting.

The Blood Transfusion Service of the Swiss Red Cross (BTS SRC) currently recommends that the RBC analysis of women in childbearing years and of patients requiring multiple transfusions are tested in addition for the blood group antigens Rhesus C, c, E, e and Kell, and for chronically transfused patients Kidd, s, S and Duffy (Fy). The Swiss hemovigilance report from 2007 showed that $13 \%$ of the reported transfusion-related adverse reactions are alloimmunizations [1]. From the cases observed in our own reference laboratory it is likely that this is only the tip of the iceberg and that the majority of the alloimmunization cases are not reported.

In Switzerland today the majority of RBC transfusions are only compatible for $\mathrm{ABO}$ and $\mathrm{RhD}$, and because the recommendations in Switzerland are not mandatory, alloimmunization in those groups mentioned above will occur. Only about $30 \%$ of the RBC concentrates produced yearly are typed for the antigens $\mathrm{Rh} \mathrm{C}, \mathrm{c}, \mathrm{E}$, e and Kell. In our opinion this situation 
should be rectified soon so that all patients requiring blood products are typed for Rhesus, Kell, Kidd, Duffy and S/s. Blood donors on the other hand should be typed for a considerably broader panel of antigens, including for instance MNS, Kell, Kidd, Duffy, Lutheran, Dombrock, Cartwright and Colton. This would not only considerably reduce the number of alloimmunizations but would serve as a platform to build up a rare donor file. We foresee the typing to be done serologically for some antigens (ABO) and molecularly, perhaps using newer methods (e.g. chip technology), for others. If future molecular technologies permit a safe, affordable and efficient mass screening, then genotyping should be the preferred approach. Such mass screening would provide the best strategy to obtain enough broadly typed RBC concentrates and may be extended to the typing of other blood components such as platelet concentrates.

The Swiss Reference Laboratory for Immunohematology at the BST SRC Berne has set up a rare donor file for Switzerland. The data in this cohort however is at present not very comprehensive and does not yet cover the whole Swiss blood donor population. We predict that, if the data collected from a broad blood group genotyping analysis are merged with the rare donor file, the supply of blood for special patients would improve dramatically. The proposed broad genotyping of blood donors must, whenever possible, include a diverse selection of ethnic minorities residing in the region covered by the blood supply. In Switzerland today, as in many other western countries, there is already a large genetic divergence in the donor population.

Despite the proposed switching from phenotype to genotype in RBC antigen tests, a patient antibody screen before transfusion will be imperative as it is not feasible to prevent all possible alloimmunizations. The reasons for this are numerous. These range from antibodies against antigens which were not analyzed to cases where it was not possible to take into account all relevant antigens in the donor and patient. Such cases often occur during emergency situations in which a fully compatible RBC may be not available. If the patient antibody screening is negative, the blood will be applied as we do now with 'type and screen'.

The strategy envisaged above would certainly bring a higher level of immunological safety in blood transfusions.
In order to improve the current transfusion practice, the new blood group genotype methods must be at least as safe as present phenotyping techniques and more cost-effective. Unfortunately, with the present technology it is difficult to judge the cost efficiency of such a proposal. The comparison should also take into account the costs occurring due to an alloimmunization which are often difficult to measure, against the extra costs sustained by a broad genotypic screening. Patient testing is not centralized and is performed in numerous hospital laboratories spread over the whole country. An electronic crossmatch between a patient and a blood product could only be done if all these laboratories are electronically linked with a central reference laboratory. In Switzerland it is unlikely that this will be implemented in the near future.

In the not too distant future it will be possible to type on the genetic level for the most antigen polymorphisms, particularly if the introduction of the recently developed chip technology is realized. But one has to take into account whether it is useful to consider all the detected polymorphisms. The clinical significance of many polymorphisms is not yet clear; thus further observations need to be done on their possible consequences.

Blood donor genotyping will in the end lead to a safer blood product supply for transfusion. A parallel development in the information technology may result in the systematic introduction of an electronic cross-match. A possible positive spin-off of this for the blood donor could be the creation of a new blood donor pass incorporating the new genotype information. In an emergency situation requiring a transfusion it should be possible to supply such donors which an optimal blood product. Blood donors are thus likely to be more motivated to donate blood, and perhaps this could lead to the recruitment of new blood donors.

\section{References}

1 Jutzi, M, Ruesch M: Annual Hemovigilance Report 2007. Bern, Swissmedic, 2008. www.swissmedic.ch/marktueberwachung/00159/00160/00437/index. html? lang=en .

2 Schonewille H, van de Watering LMG, Loomans DSE, Brand A: Red blood cell antibodies after transfusion: factors influencing incidence and specificity. Transfusion 2006:46:250-256. 